\title{
Elucidating the Influence of Sulfur Vacancies on Nonradiative Recombination Dynamics in $\mathrm{Cu}_{2} \mathrm{ZnSnS}_{4}$ Solar Absorbers
}

Zhi Chen, ${ }^{1}$ Ping-Zhi Zhang, ${ }^{1}$ Yu Zhou, ${ }^{1}$ Xingming Zhang, ${ }^{1}$ Xiaorui Liu, ${ }^{2}$ Zhufeng Hou, ${ }^{3}$ Jianfeng Tang, ${ }^{1 *}$ Wei $\mathrm{Li}^{1 *}$

${ }^{1}$ School of Chemistry and Materials Science, Hunan Agricultural University, Changsha 410128, People's Republic of China

${ }^{2}$ School of Chemistry and Chemical Engineering, Southwest University, Chongqing 400715, People's Republic of China

${ }^{3}$ State Key Laboratory of Structural Chemistry, Fujian Institute of Research on the Structure of Matter, Chinese Academy of Sciences, 350002, Fuzhou, China

Corresponding authors:

weili@ hunau.edu.cn (W.L.); jftang @ hunau.edu.cn (J.F.T.) 
Table S1. Averaged atom displacement velocity $(\AA / \mathrm{fs})$ for pristine and defective CZTS systems. Data presented in the Table are magnified by 1000 .

\begin{tabular}{cccc}
\hline System & Pristine & $V_{S}^{0}$ & $V_{S}^{2+}$ \\
\hline Velocity & 3.63 & 4.34 & 4.52 \\
\hline
\end{tabular}

Table S2. Standard Deviation (SD, in unit of eV) of orbital energies for pristine and defective systems.

\begin{tabular}{|c|c|c|c|c|c|c|c|}
\hline & \multicolumn{2}{|c|}{ Pristine } & \multicolumn{2}{|c|}{$V_{S}^{0}$} & \multicolumn{3}{|c|}{$V_{S}^{2+}$} \\
\hline Orbital & VBM & CBM & VBM & CBM & VBM & Trap & CBM \\
\hline SD & 0.02146 & 0.05580 & 0.03168 & 0.05582 & 0.02237 & 0.02535 & 0.05603 \\
\hline
\end{tabular}



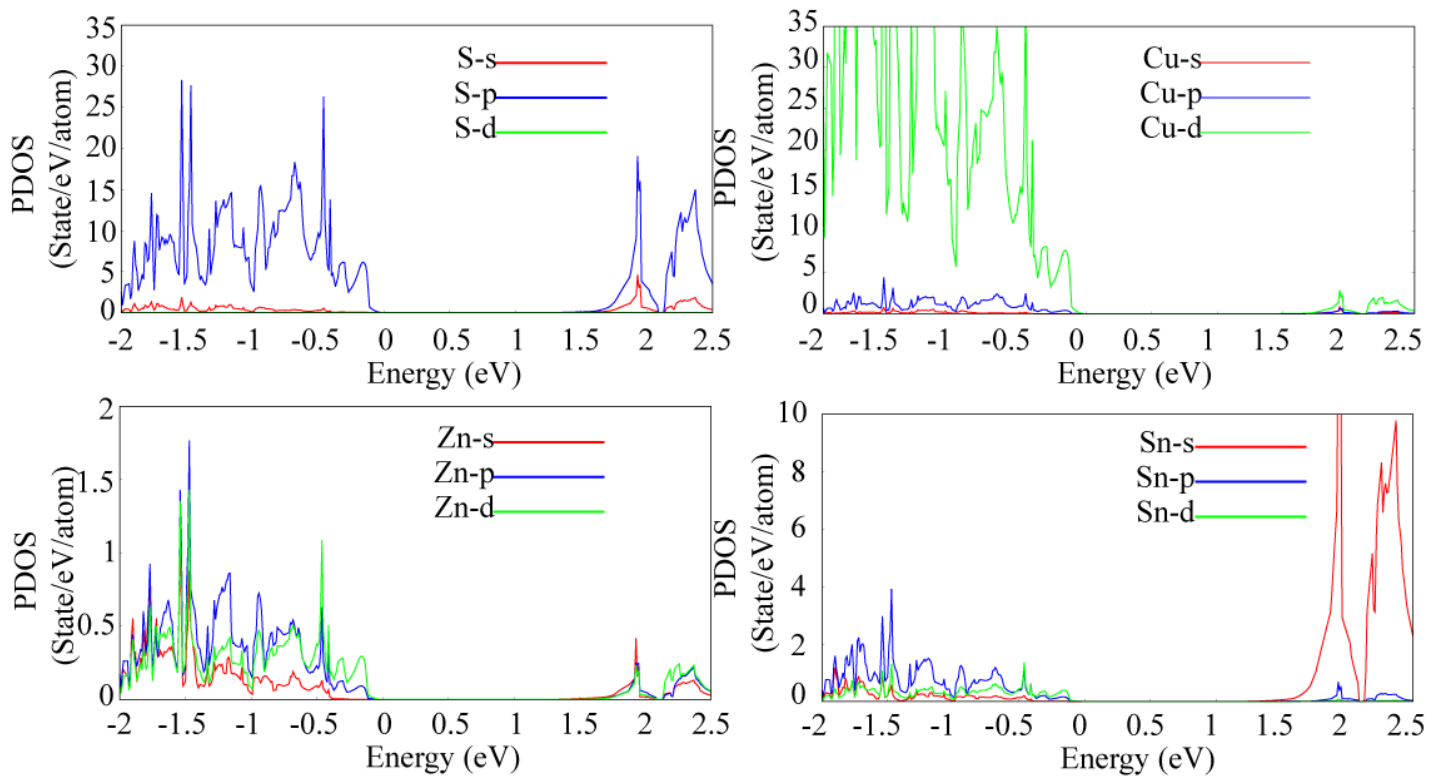

Figure S1. Orbital-resolved density of states for pristine system.
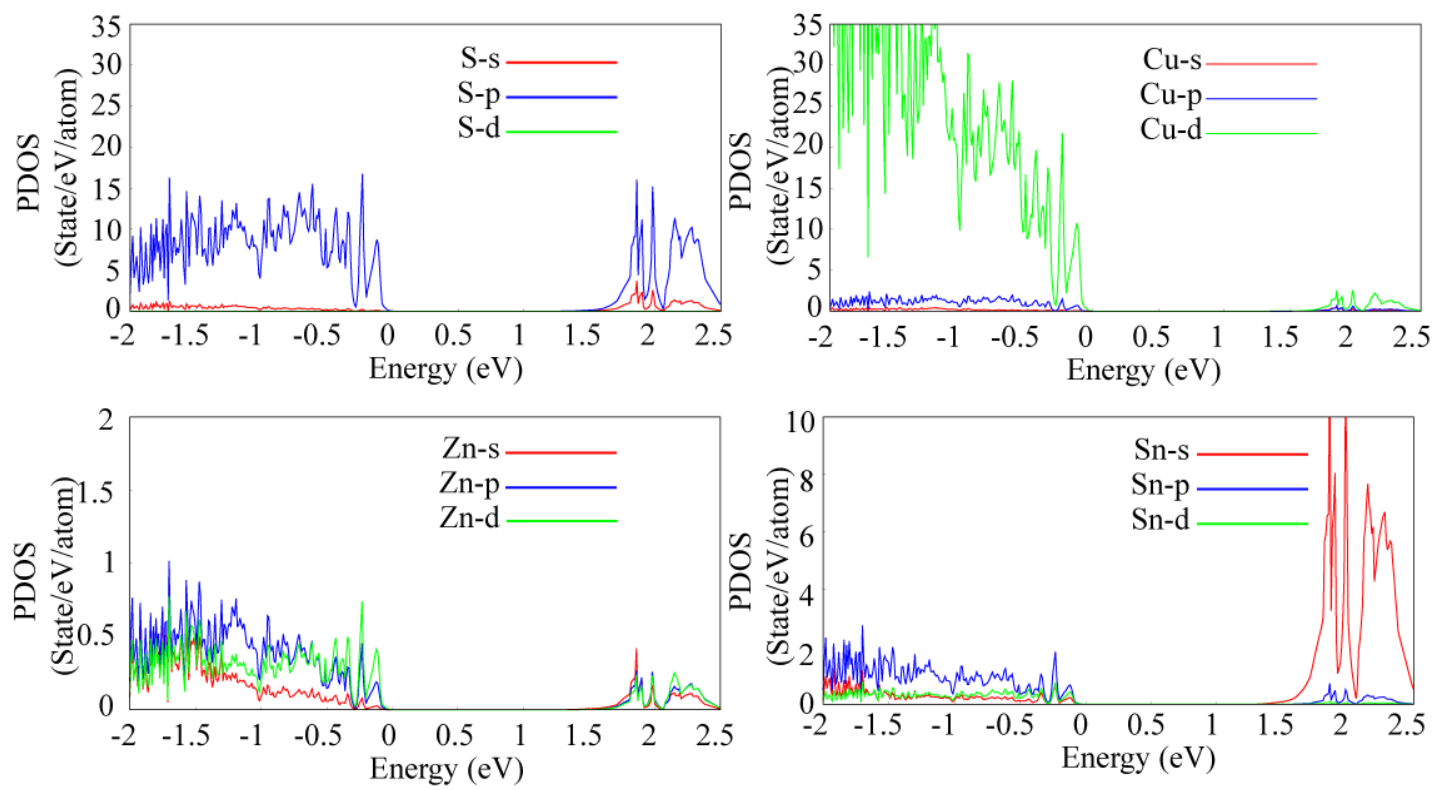

Figure S2. Orbital-resolved density of states for $V_{S}^{0}$ system. 

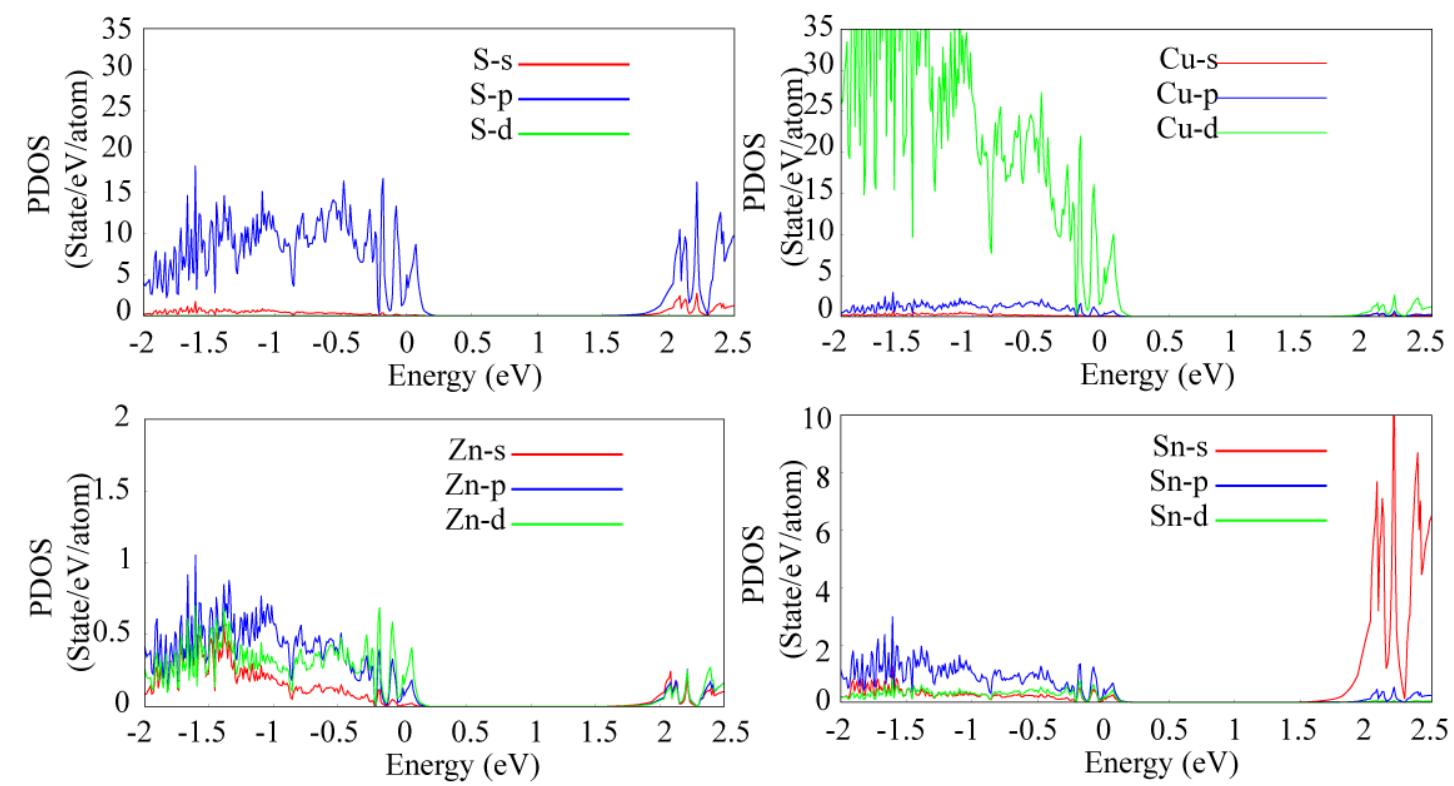

Figure S3. Orbital-resolved density of states for $V_{S}^{2+}$ system.
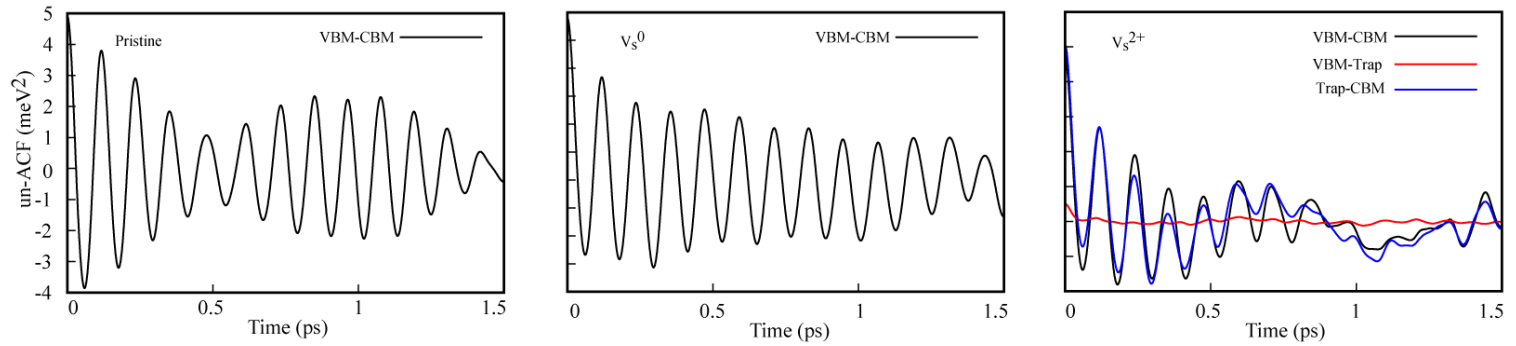

Figure S4. The un-normalized autocorrelation functions (un-ACF) of the gap fluctuation between VBM and CBM in CZTS with pristine and neutral $V_{S}^{0}$; VBM and CBM, VBM and trap, $\mathrm{CBM}$ and trap in $V_{S}^{2+}$. Initial value represents the squred standard deviation of gap flucuation. 

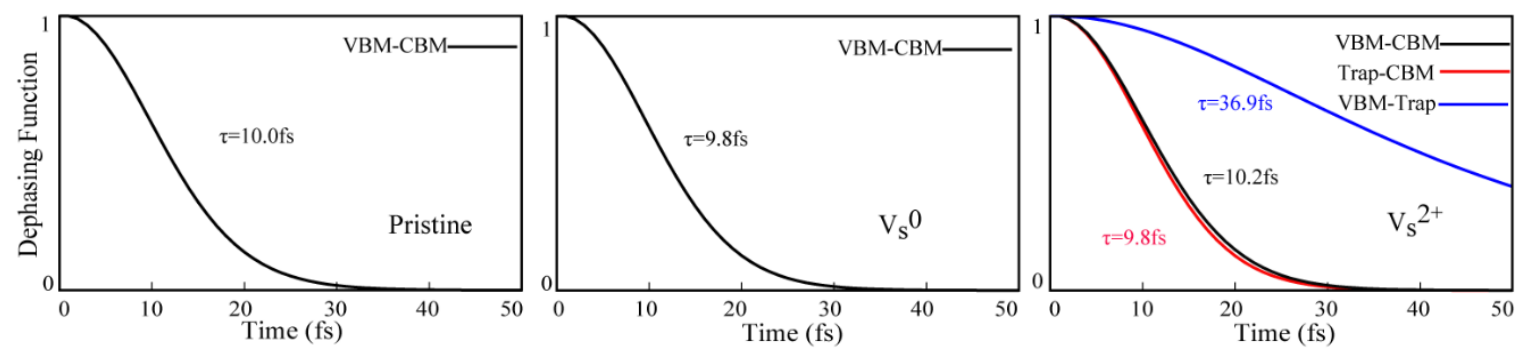

Figure S5. The dephasing functions of pristine and defective CZTS systems. 